\title{
Collaborative (One Health) approaches to address emerging antimicrobial resistance
}

\author{
Lloyd Reeve-Johnson* \\ Professor of One Health, Faculty of Science, Health, Education and Engineering, University of Sunshine Coast, Maroochydoore, Queensland, Australia
}

The concept of 'One Health' recognizes that the health of people is clearly connected to the health of animals and the environment and encourages multidisciplinary collaboration locally, nationally, and globally to achieve better health. The United States Center for Disease Control (CDC) states that six out of ten infectious diseases in humans are spread from animals [1]. This statement describes half the picture. It omits the corollary that animals are also infected by humans. This is an important perspective when tackling organisms not susceptible to antimicrobial treatment. Multiple species provide reservoirs for pathogens to survive, replicate and opportunistically infect other species. If the problem of antimicrobial resistance is to be successfully addressed a holistic, multi-species approach is critical. To cite an earlier paper, "The human may be the most valued species to some, yet vectors, pathogens and climatic conditions have no such pretensions. Knowledge of host-pathogen interactions with each species provides new perspectives and possibilities for comprehensive solutions" [2].

The increased incidence of organisms resistant to multiple classes of antimicrobial agent is of major concern for the future health of human and animals. Sources attribute 700,000 additional human deaths to problems associated with antimicrobial resistance at present and forecast these to rise to up to 10 million additional deaths per year by 2050 [3]. The causes rest with usage patterns and the volumes of antimicrobials being used both in human and veterinary medicine. As the magnitude and significance has become clearer, there has been a trend for finger pointing. In November 2016, an open letter endorsed by the Royal Society of Medicine, Faculty of Public Health and British Medical Association called for an immediate UK-wide ban on routine mass use of antimicrobials in animals. This sentiment is neither new nor is there clear evidence at present that a ban focused exclusively on veterinary uses would significantly impact the emergence of antimicrobial resistance in humans. There are a number of factors that drive demand for antimicrobials in different ways in human an veterinary health which need to be considered, these include:

1. Cost: In most OECD countries antimicrobials are free in hospitals and subsided in general practice so there is little cost constraint on human supply. In contract in veterinary medicine, the owner generally has to pay. Thus antimicrobial use is cost-constrained and in commercial agriculture where the greatest volumes are used, benefits have to outweigh cost.

2. Usage patterns favouring mass selection of resistant organisms: Human use in hospitals is in the face of nosocomial infections, in a transitory population. Bed-pressure compels people to return to their community whilst still treating infections acquired within hospitals while others enter the hospital 'herd' bringing new bacterial loads from the community. Most commercial agricultural units (particularly poultry and pigs units) have strict biosecurity measures which limit transfer of organisms between the herd and external environment and in particular to humans.

3. Usage patterns favouring specific selection of resistant organisms: Routine human use of antimicrobials includes common infections such as urinary tract infections, upper respiratory tract infections, orthopaedic prophylaxis and travel prophylaxis in the general population. In veterinary medicine overuse in companion animals for dermatological conditions and minor infection is also relevant given the close proximity of these animals with humans.

4. Likelihood of geographical dissemination of resistant organisms: The propensity of humans to travel including across international borders is greater than at any time in history. Human biosecurity measures are very lax compared to animal movement restrictions. Bird migration has also been studied although risk of introduction of a resistant human pathogen at adequate dose into the human population is considerably less than from a human traveller carrying the same organism.

5. Proximity between treated animals and humans. Particularly in developing nations animals and humans often live in close proximity. Developing nations and areas of conflict with poor health infrastructure are the source of the majority of emerging diseases. They also represent areas of high risk for antimicrobial resistance if antimicrobials are used indiscriminately in any species.

6. Likelihood of direct transmission of resistant organisms. Resistant organisms are far more likely to be spread directly to another human than from an animal to a human. In part this reflects normal social contact and in part pathogen-host preferences.

7. Total volume of antimicrobials used. As populations increase greater volumes are used. Antibiotic residues also leach into run-off causing environmental burden and selection pressure for resistant organisms.

The consequence of continued loss of antimicrobial susceptibility would be vastly more complex to manage than even the well publicised 2014-2015 Ebola virus crisis. Yet, analysis of the challenges of the Ebola crisis revealed that no single organization could respond as

Correspondence to: Lloyd Reeve-Johnson, Professor of One Health, Faculty of Science, Health, Education and Engineering, University of Sunshine Coast, Maroochydoore, Queensland, Australia, E-mail: lloyd@goydpark.com

Key words: antimicrobial resistance, one health, healthcare economics, translational research

Received: February 14, 2017; Accepted: February 23, 2017; Published: February 25, 2017 
comprehensively as was required [4] and effective controls could not be implemented in the absence of a multidisciplinary approach to managing epidemiology, surveillance, laboratory, clinical cases, funerals, logistics and security, social mobilization and health education, psychosocial support, and other key functions. In the case of widespread antimicrobial resistance, potentially every hospital in every country would have major issues and every contact between man, animals and the environment would continue to transfer resistant organisms. Organisms currently easily treated could become potent pathogens.

Antimicrobial resistance is a critical area for interdisciplinary and international collaboration and preparedness. However, to succeed with multidisciplinary One Health type collaborations there are some key prerequisites of which we should be mindful [2]:

1. There should be no hierarchy between contributors: doctors, veterinarians, engineers, economists or others all meet on an equal footing as specialists in narrow fields collaborating to create broad solutions that are practical to implement and sustainable. None should be viewed in the One Health group as having ownership as this quickly undermines motivation of others.

2. One Health agendas should be driven by agreed measurable clinical end-points.

3. Business administration, cost management and commercialization are vital skill components to ensure sustainability.

4. The human may be the most valued species to some, yet, vectors, pathogens and climatic conditions have no such pretentions Knowledge of host-pathogen interactions with each species provides new perspectives and possibilities for comprehensive solutions.

5. Accurate joint communication efforts in journals, at conferences and to media and politicians is more helpful than segregating research into human versus animal components.

6. Multi-species epidemiological and disease transmission studies are more likely to lead to a sustainable solution than focusing on the human component alone.

7. Joint development, evaluation and utilisation of new diagnostic methods, medicines and vaccines will lead to better prevention and control of diseases if applied across multiple species.

8. Conclusions should have a sound breadth of evidence (preferably multispecies over time) to support them.

It has been demonstrated [5] that provision of healthcare in both the human and veterinary sectors depends upon a series of transactions between a relatively small number of core stakeholders and a multitude of external influences including belief systems which do not necessarily relate directly to the immediate healthcare need. The participants in healthcare interact and rationalize perspectives of utility through a form of economic exchange which is constantly evolving to greater complexity to cater to increasingly diverse influences. In all forms of healthcare demand generally pre-exists. Education, awareness, media constructs and supply of treatments influence the way new products and interventions are utilized. Central to healthcare provision and uptake of antibiotic use however is the individual adoption process driven by the patient (or in veterinary care the animal owner), which vitally includes the initial perception of illness, the decision to seek treatment and diagnosis, setting up the treatment plan and then the delivery of the treatment with subsequent evaluation of the effectiveness of the treatment plan which in turn impacts compliance [5]. Each of these steps is a potential point of influence and can be used to influence the type of treatment. Figures 1 and 2 demonstrate how antimicrobial uptake is influenced at each stages of the decision process in a way that is equally applicable to human and veterinary healthcare. Education, marketing, cost constraint, regulation and health literacy are all factors

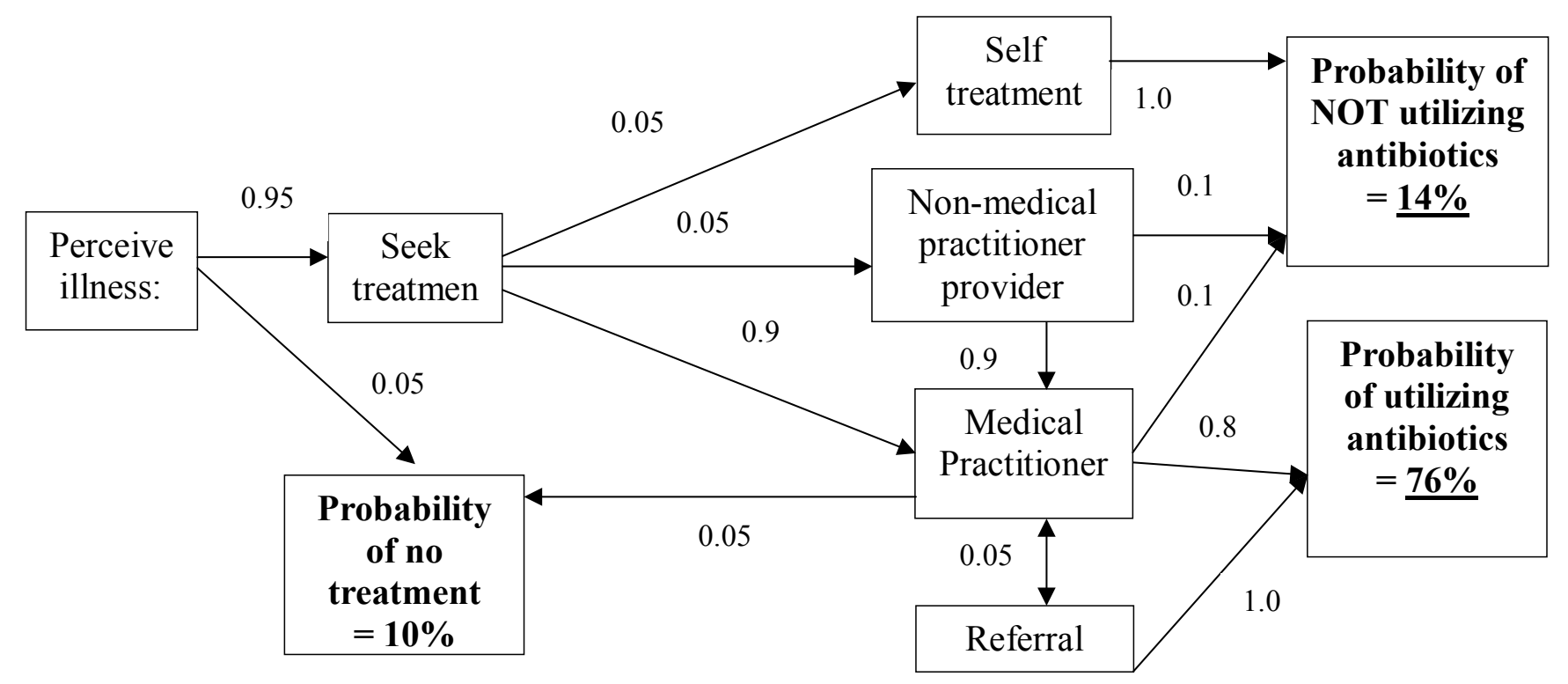

Figure 1. Illustration of a typical decision cascade illustrating likely antimicrobial use in general medical practice. 


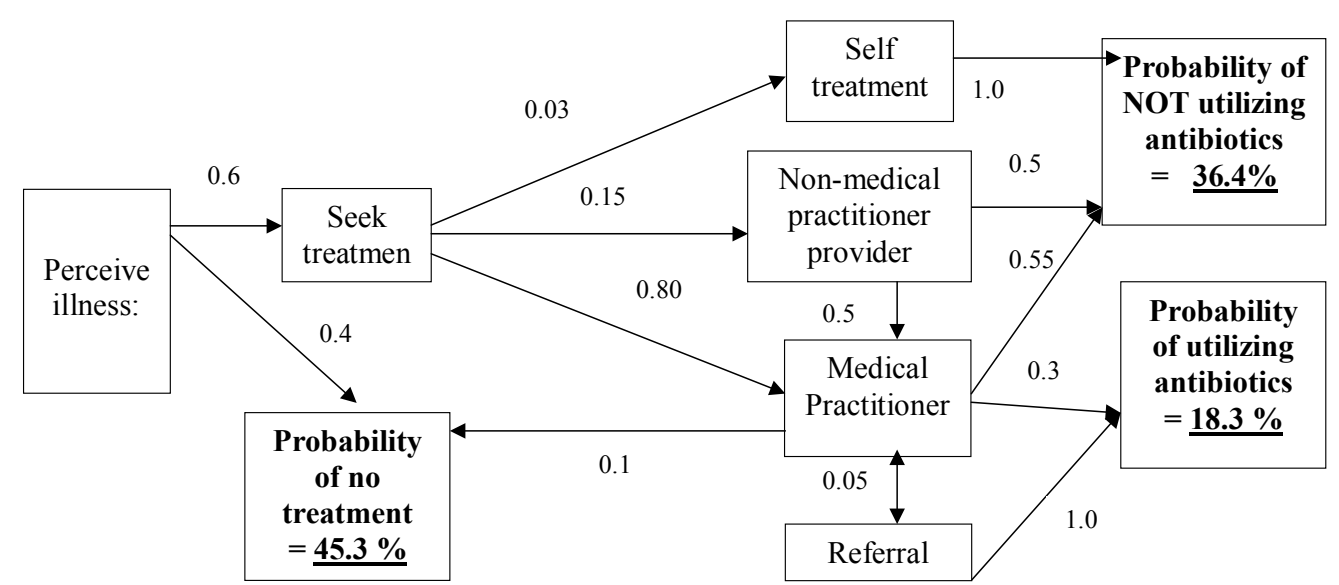

Figure 2. Illustration of an 'influenced' decision cascade showing how antibiotic use can be greatly reduced via altering sequential treatment decisions.

relevant to altering outcome and way antimicrobials are used.

\section{References}

1. https://www.cdc.gov/onehealth/

2. Reeve-Johnson L (2016) Forensic science and applications to One Health collaborations. Chapter in Practical Veterinary Forensics, Bailey D (Ed.), CABI publishing, UK
3. Gibbens N (2017) Vets and antimicrobials. Veterainry Record 1: 7.

4. Cooper C,Fisher D,Gupta N,MaCauley R,Pessoa-Silva CL (2016) Infection prevention and control of the Ebola outbreak in Liberia, 2014-2015: key challenges and successes. BMC Med 14: 2. [Crossref]

5. Reeve-Johnson L (2017) Applying game theory to One Health: Modelling Human Healthcare Delivery. Intl Anim Health J May 2017 (in press).

Copyright: (2017 Reeve-Johnson L. This is an open-access article distributed under the terms of the Creative Commons Attribution License, which permits unrestricted use, distribution, and reproduction in any medium, provided the original author and source are credited. 